http://dx.doi.org/10.4314/bajopas.v11i1.25S

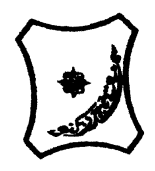

Bayero Journal of Pure and Applied Sciences, 12(1): 152 - 155

ISSN 2006 - 6996

\title{
VULVOVAGINAL CANDIDASIS AMONG FEMALE PATIENTS ATTENDING YUSUF DANTSOHO MEMORIAL HOSPITAL AND BARAU DIKKO SPECIALIST HOSPITAL KADUNA
}

\author{
*Aliyu, M.Y., Obajemu, O., Aliyu, A.M. and Gabriel, R. \\ Department of Applied Science C.S.T. Kaduna Polytechnic Kaduna \\ *Corresponding author maryamt2000@gmail.com. +2348034533924
}

ABSTRACT

Vulvovaginal candidiasis (VVC) is a major cause of lower genital infections in women, especially in developing countries and cause significant morbidity and financial burden on the already weak economy. The objective was to isolate and identify Candida spp responsible for Vulvovaginitis. Two hundred (200) vaginal swabs were collected from female patients 15years and above, attending Yusuf Dantsoho Memorial Hospital (YDMH) and Barau Dikko Specialist Hospital (BDSH) all within Kaduna metropolis. The samples were analysed for the presence of Candida spp using standard procedures of microscopy, culture and biochemical identification. The overall incidence of VVC was $79.5 \%$ (159/200), with higher incidence among patients attending Yusuf Dantsoho Memorial Hospital 84.8\% (117/138), than Barau Dikko Memorial Hospital 67.7\% (42/62). Four (4) yeast species were isolated and identified which include Candida krusie, Candida parapsilosis and Candida glabrata. C. krusie had the highest percentage occurrence of 42.5\% (39/159), while $C$. parapsilosis had the least percentage occurrence of $11.3 \%$ (18/159). Higher incidence was recorded among age group 40 and above 92.8\% (13/14) and lowest among age group 31-40 76.5\% (39/51). Highest incidence was also recorded among widow 100\% (18/18) and lowest among married $76.8 \%$ (109/142). The high percentage of positive samples is an indication that there is a high incidence of candidiasis among the study population. There is need for regular screening of women for VVC and other sexually transmitted infections.

Key words: Incidence, Vulvovaginal Candidiasis, Isolation, Kaduna.

\section{INTRODUCTION}

Vulvovaginal candidiasis (VVC) is a fungal infection of the female lower genital tract, the vulva and the vagina, caused by Candida species (Sobel, 2003). It is also known as candidiasis or moniliasis. This infection is characterized by intense vulva and vaginal irritation (itching) which often give rise to inflammation, soreness, redness and white spots in and around the genital tract. Thick, cheesy white, curd-like vaginal discharge generally adherent as white patches to the vaginal wall, cervix and labia minora is observed. Also, painful urination and painful intercourse are associated with the infection. The infection could be acute, recurrent or chronic. Interestingly, most if not all women carry Candida in the vagina as normal flora at some points in their lives, yet without symptoms or signs of vaginitis and usually with a low concentration of yeast organism (Heidrich et al., 1984).

Globally, VVC remains a common problem affecting all strata of society (Ugwa, 2015). An estimated $75 \%$ of women will experience at least an episode of VVC during their life time (Singh, 2003). Diagnosis of VVC based solely on patients history and genital examination is not possible because of the low specificity of symptoms and signs as other causes mimic VVC like leukorrhoea (vaginal discharge) and pruritus vulvae (vulva itching) (Onuora et al., 2018).

Candida albicans is both the most frequent colonizer and responsible for most cases of VVC. Nevertheless, there have been reports demonstrating an increment in the frequency of cases caused by non-albicans species with Candida glabrata consistently being the leading species (Maceato and Kaufman, 1991).

The objective of this paper was to isolate and identify Candida Spp responsible for Vulvovaginal candidiasis, determine the incidence rate of Vulvovaginal Candidiasis and determine the relationship between demographic factors and the occurrence of Vulvovaginal Candidiasis among female patients attending Yusuf Dantsoho Memorial Hospital and Barau Dikko Specialist Hospital Kaduna, Nigeria. 


\section{MATERIALS AND METHODS Study Area}

The study was conducted in two different hospitals, Yusuf Dantsoho Memorial Hospital Tudun Wada and Barau Dikko Specialist Hospital all within Kaduna Metropolis, Kaduna State, Nigeria. The study sites are the two major tertiary health facilities that serves mainly patients from within and out site Kaduna metropolis.

\section{Study Design}

The study was a hospital based cross sectional study, comprising female patients aged 15years and above attending the two selected hospitals.

\section{Ethical Clearance}

Ethical clearance was obtained from the ethical committee of the Kaduna State Ministry of Health. The selected hospitals were visited to discuss the feasibility of the study and the cooperation of the management, clinicians and laboratory staffs prior to the commencement of the work.

\section{Determination of Sample Size}

The minimum sample size for the study was estimated using the fischer's formula using a prevalence rate of $11 \%$ as demonstrated by Sobel et al (2008).

$\mathrm{n}=\mathrm{Z}^{2} \mathrm{Pq}$

Where

$\mathrm{n}=$ minimum sample size

$Z$ =normal standard deviation for the required level of confidence.

$\mathrm{P}=$

$q=1.0-p$

$\mathrm{L}=$ allowable error at $5 \%=0.05$

$\mathrm{Z}=1.96$

$P=11 \%=0.11$

$q=1.0-0.11=0.89$

$\mathrm{L}=0.05$

$\mathrm{n}=\frac{1.962 \times 0.11 \times 0.89}{0.052}$

$$
0.052
$$

$\mathrm{n}=150$. However this was rounded to the nearest 100 to give 200 .

\section{Sample Collection}

A total of two hundred (200) vaginal swabs were collected using sterile cotton swab stick. The vaginal/vulva swabs were collected with the help of experienced hospital personnel. These were done by inserting the swab into the vaginal gently rotated for 10-30 seconds in the vagina. The swab was then withdrawn gently ensuring that it does not touch the skin and immediately inserted aseptically into the swab package, this procedure was carried out according to the method by Onuorah et al (2018).

\section{Isolation of Candida spp}

Each of the vaginal swabs was inoculated on fresh sterilized sabouraud dextrose agar (SDA) plates prepared aseptically and incubated at room temperature before they were observed for growth. The suspected colonies of interest with distinct cultural characteristics developed after 72 hours of incubation. The colonies were purified by repeated sub culturing on sterile SDA and later stored on sterile SDA slants for further test.

\section{Microscopic Examination of the Sample}

About $1 \mathrm{ml}$ of normal saline was put in the tube containing the swab to cover the cotton bud, shaken and allowed to stand for some minutes. A drop of this was placed on a clean greasefree slide and was viewed with low power objectives (10x and $40 x$ ) for yeast cells as described by Uzor et al. (2016).

\section{Biochemical Test}

The plates that showed pure culture were used for the biochemical identification tests, which include sugar assimilation (glucose, maltose, sucrose and lactose) and urea utilization. The procedure of Menza et al (2013) was used in carrying out the test. The carbohydrates impregnated filter paper discs were placed on sterile carbohydrate-free yeast nitrogen base agar contained in petri dishes and incubated at $30^{\circ} \mathrm{C}$ for 18hours. The presence of growth around the carbohydrate-free .paper disc was there after observed as an indication of the ability of the isolate to assimilate a sugar. While urea agar slant was used for urea utilisation, where it was streaked with the test isolate and incubated at $37^{\circ} \mathrm{C}$ for 48 hours and observed for colour change from light orange to bright pink, which was an indication that urea has been utilised.

\section{RESULTS AND DISCUSSION}

Out of 200 samples analysed microscopically 159 sample were positive for candidiasis while 41 were negative, giving an overall incidence rate of $79.5 \%$. This result agrees with $81.5 \%, 84.5 \%$ and $84.5 \%$ reported by Onifade and Olorunfemi, (2005) in Ondo State, Ibrahim et al. (2009) in Kano and Ugwa, (2015) in Aminu Kano Teaching Hospital, North-West Nigeria. The result is however slightly higher than $61.8 \%$ and $62.2 \%$ reported by Akpan et al. (2011) in Uyo and Onuora et al. (2018) in Enugu State. The high incidence of VVC (79.5\%) is an indication of candidiasis among female patients attending Yusuf Dantsoho Hospital and Barau Dikko Hospital, Kaduna. 
Special Conference Edition, November, 2019

Table 1: Overall incidence of Vulvovaginal Cadidiasis amongst Female Patients Attending Yusuf Dantsoho and Barau Dikko Hospital No of samples collected No positive (\%) No negative (\%) 200

$159(79.5)$
41(20.5)

Four (4) yeast types, namely Candida albicans, Candida Krusei Candida parapsilosis and Candida glabrata were identified, with percentage frequency of occurrence of 24.5, 42.1, 11.3 and 22.0 respectively. Of the four yeast types, Candida albican, Candida Krusei and Candida glabrata were also isolated by Emele, (2010), who reported Candida albicans being the second most prevalent among the yeast species isolated. This agrees with our findings. Even though Candida albicans was not the most prevalent yeast specie isolated in this research, the relatively high incidence of Candida albicans in this work could be due to the tolerance of this yeast species to acid conditions found in the vagina due to vaginal secretions and type of underwear worn by women.

Table 2: Frequency Occurrence of Yeast isolated from the Vaginal Swabs Sample.

\begin{tabular}{lll}
\hline Yeast identified & $\begin{array}{l}\text { No } \\
\text { occurrence }\end{array}$ & $\begin{array}{c}\text { of } \\
\text { frequency }\end{array}$ \\
\hline & & \\
Candida albicans & 39 & 24.5 \\
Candida krusei & 67 & 42.1 \\
Candida parapsilosis & 18 & 11.3 \\
Candida glabrata & 35 & 22.0 \\
Total & $\mathbf{1 5 9}$ & $\mathbf{1 0 0}$ \\
\hline
\end{tabular}

The incidence of vulvovaginal candidiasis was found to be higher at Yusuf Dantsoho Hospital, Tudun wada Kaduna (37.6\%) than at Barau Dikko Hospital with an incidence of $26.4 \%$ this variation could be due to the fact that most of the women patients attending Yusuf Dantosho
Hospital have low level of awareness on personal hygiene while those attending Barau Dikko specialist Hospital have awareness about personal hygiene and changing of underwear at regular intervals, considering the location of the two hospital.

Table 3: Incidence of Vulvovaginal Candidiasis with Respect to the Hospitals Evaluated.

\begin{tabular}{llll}
\hline Hospital & No Screened & No Positive & \% Positive \\
\hline Yusuf Dantsoho Memorial Hospital & 138 & 117 & 84.8 \\
Barau Dikko Specialist Hospital & 62 & 42 & 67.7 \\
Total & $\mathbf{2 0 0}$ & $\mathbf{1 5 9}$ & $\mathbf{7 9 . 5}$ \\
\hline
\end{tabular}

Highest incidence was found among women age group above 40 years and lowest among age group 31-40. This could be due to the fact that there could be other possible risk factors that might predispose women to the disease other than being sexually active, such as diabetes, type of under wear and use of antibiotics. This report is not in accordance with the reports of Ibrahim et al. (2009), Akpan et al. (2011), Emeribe et al. (2015) and Ugwa, (2015), who reported highest incidence among sexually active women.

Table 4: Incidence of Vulvovaginal Candidiasis Infection with Respect to Age Among Women Attending the Yusuf Dantsoho and Barau Dikko Hospitals, Kaduna.

\begin{tabular}{llll}
\hline Age group & No Screened & No Positive & \% Positive \\
\hline below 20 & 28 & 24 & 85.7 \\
$20-30$ & 107 & 83 & 77.5 \\
$31-40$ & 51 & 39 & 76.5 \\
above 40 & 14 & 13 & 92.8 \\
Total & $\mathbf{2 0 0}$ & $\mathbf{1 5 9}$ & $\mathbf{7 9 . 5}$ \\
\hline
\end{tabular}

The incidence was highest among widows and lowest among married women. This is in contrast to the reported work of Ugwa, (2015) who reported highest incidence among married women compared to unmarried women. Akpan, et al. (2011) in which the incidence of VVC was higher in singles than in married women and Ibrahim et al. (2009) who reported higher incidence in unmarried women compared to married women. 
Special Conference Edition, November, 2019

Table 5: Incidence of Vulvovaginal Candidiasis Infection with Respect to Patients Marital

Status

\begin{tabular}{llll}
\hline Marital status & No Screened & No Positive & \% Positive \\
\hline Single & 13 & 11 & 84.6 \\
Married & 142 & 109 & 76.8 \\
Divorced & 27 & 21 & 77.8 \\
Widow & 18 & 18 & 100 \\
Total & $\mathbf{2 0 0}$ & $\mathbf{1 5 9}$ & $\mathbf{7 9 . 5}$ \\
\hline
\end{tabular}

\section{CONCLUSION}

The high percentage of positive samples $(79.5 \%)$ is an indication that there is a high incidence of candidasis among the study population. Four yeast species Candida albicans, Candida krusei, Candida glabrata and Candida parapsilosis were found to be associated with vulvovaginal Candidiasis among the study population. High incidence was reported in Yusuf Dantshoho hospital $84.8 \%$ compared to Barau Dikko specialist Hospital (67.7\%). Age and

\section{REFERENCES}

Akpan, C.E., Ekpenyong, J.E., and Ibu, J.O. (2011). Incidence of Vulvovaginal Candidiasis among Women in Tight Fitting Underwears: The Need for Counselling and Health Education. 3(10): $478-481$.

Emele, F.E. (2010). Vulvovaginal Candidiasis in Nigeria: Increasing Importanc of Non Albicams Candida. 10 ${ }^{\text {th }}$ ASM Conference on Candida and Candidiasis, Miami FI, USA, ABS 194B.

Emeribe, A.U., Nasir, I.A., Onyla, J. and Ifunanya, A.L. (2015). Prevalence of Vulvoviginal Candidiasis among Non-pregnant Women Attending a Tertiary Health Care Facility in Abuja, Nigeria. Research and Reports in Tropical Medicine, 5(6):37-42.

Heidrich, F.E., Berg, A.O. and Bergman, J.J. (1984). "Clothing Factors and Vaginitis. Journal of family practice. 19:491- 494.

Ibrahim, S.A., Ugwa, E.A., Agu, O.C. and Onuorah, C.C. (2009). Prevalence of Vulvovaginal Candidiasis at a Gynaecological Clinic in Kano, North-West Nigeria. Borno Medical Journal, (1):1-4.

Maceato, M.L. and Kaufman, R.H. (1991). Fungal Vulvovaginitis. Current Journal of Gynecology 3: $52-849$.

Menza, N., Wanyoike W., and Muturi M. (2013). Prevalence of Vaginal Candidiasi and Determination of the Occurrence candida species in Pregnant Women Attending the Ante-natal Clinic of Thika District Hospital, marital status also seems to play a significant role in the acquisition of Candidiasis,

\section{RECOMMENDATIONS}

1. Regular screening of women for VVC and other sexually transmitted infections should be carried out.

2. Further work on the involvement of bacteria and protozoa in vaginal infection is encouraged to have comprehensive information about nature of infection of women reproductive system.

Kenya. Open Journal of Medical Microbiology. 3(4):1-9.

Onifade, A.K. and Olorunfemi, O.B. (2005). Epidemiology of Vulvoviginal Candidiasis in Female Patients in Ondo State Government Hospital. Journal of Food, Agriculture and Environment, 3:118-119.

Onoura, C., Ndibe, O. and Nzeribe, O. (2018). Assessment of Vulvovaginal Candidiasis During Pregnancy in Enugu State, Nigeria. African Journal of Nursing and Midwifery, 6(3):762-767.

Singh, S.I. (2003). Treatment of Vulvoginal Candidiasis. Clinical Review, 36(9):26-30.

Sobel J.D. (2003). Management of Patients with Recurrent Vulvovaginal Candidiasis. Drugs 63: 1059 - 1066.

Sobel J.D, Faro S, Force R.W. (2008). Vulvovaginal Candidiasis: Epidemiologic, Diagnostic and Therapeutic Considerations. American Journal of Obstetrics and Gynecology, 178(2):203-211.

Ugwa, E.A. (2015). Vulvovaginal Candidiasis in Aminu Kano Teaching hospital North-West Nigeria: Hospital-Based Epidemiology Study. Annals of Medical and Health Sciences Research, 5(4):274-278.

Uzzoh C.V. Iheukwumere I.H Umezurike K.C Onyewenjo S.C. (2016). Prevalence of Candida albicans among Women Attending Federal Medical Centre Asaba, South-South, Nigeria. Advances in life Science and Technology, 6:52-58. 